\title{
Theoretical Analysis and Simulation of High Pressure Water Jet Cleaning Process
}

\author{
Shuce Zhang ${ }^{1}$, Xueheng Tao ${ }^{*}{ }^{* 1,2}$, Jinshi $\mathrm{Lu}^{1,2}$, Xuejun Wang ${ }^{1,2}$, Zhenhua Zeng ${ }^{3}$ and Xiaofei Pang ${ }^{4}$ \\ ${ }^{1}$ School of Mechanical Engineering and Automation, Dalian Polytechnic University, Dalian, 116034, China \\ ${ }^{2}$ National Engineering Research Center of Seafood, Dalian, 116034, China \\ ${ }^{3}$ Dalian Modern Auxiliary Machines Development and Manufacturing Co. Ltd, Dalian, 116600, China \\ ${ }^{4}$ Inner Mongolia Eppen Biotech Co. Ltd, Chifeng, 024070, China
}

\begin{abstract}
Continuous high pressure water jets currently have many industrial applications, for example, mining coal, cutting materials, and cleaning fouling layers. To gain a thorough understanding of the helix nozzle structure, which is optimized by adding an internal spiral line from a cylindrical contracting nozzle, and thereby study jetting characteristics and cleaning effectiveness, a numerical simulation method is presented to capture the features of velocity magnitude and directions and the dynamic pressure in and out of the nozzle. This approach follows the fact that it is more efficient to perform shearing action on the fouling layer in the cleaning process beyond an axial impacting action functioned by a traditional cylindrical contracting nozzle. The results reported in this paper show that the velocity magnitude of the jet issuing from the helix nozzle produces a relatively concentrated distribution, considering the guidance and disturbance by the spiral line, and the dynamic pressure shows similar characteristics. The shearing action of the tangential jetting and the entrainment effect of the shear eddy strengthen cleaning effectiveness.
\end{abstract}

Keywords: Dynamic pressure, fouling layer, helix nozzle, jet cleaning, water jet, Magnitude

\section{INTRODUCNTION}

Cleaning, the removal of fouling layers from a material surface, is a significant operation in industrial production. A variety of technologies with years of development are available to improve the high-pressure water jet cleaning process. Robert W. Wild and Joseph G. Keeney (1978) invented a high-pressure jet wall cleaner apparatus that is capable of efficiently washing contaminated walls in a nuclear reactor by confining high-pressure water sprays and the turbulent mist created thereby within a housing to avoid environmental contamination. M. C. Leu et al. (1998) presented a mathematical model of a stationary water jet cleaning process in which the cleaning width - as a function of standoff distance, water pressure, and nozzle radius-is derived by considering the structure of the water jet and the cleaning mechanism [1]. L. Gensheng et al. (2007) investigated the production-enhancing mechanisms of deep penetration perforation with a high-pressure water jet and discussed its feasibility in improving formation fracturing and acidizing treatment [2]. Guha et al. (2011) assessed pressure distribution on the cleaning surface and performed an experimental study to identify the pressure characteristics [3].

*Address correspondence to this author at the School of Mechanical Engineering and Automation, Dalian Polytechnic University, Dalian, 116034, China; Tel: 86-411-86323773; Fax: 86-411-86323786; Email: xhtao@dlpu.edu.cn
Focusing on the helix nozzle structure presented by S. Zhang et al. [4] as shown in Fig. (1), this paper extends their work to perform a detailed analysis of jetting performance guided by the spiral line and cleaning efficiency. Continuous interaction of the spiral line with the cylindrical contracting structure resulting in the flow of the water jet is complex and different from that issuing from an axisymmetric structure nozzle. To bypass theoretical limitations, a description of the flow characteristics is presented based on the simulation results.
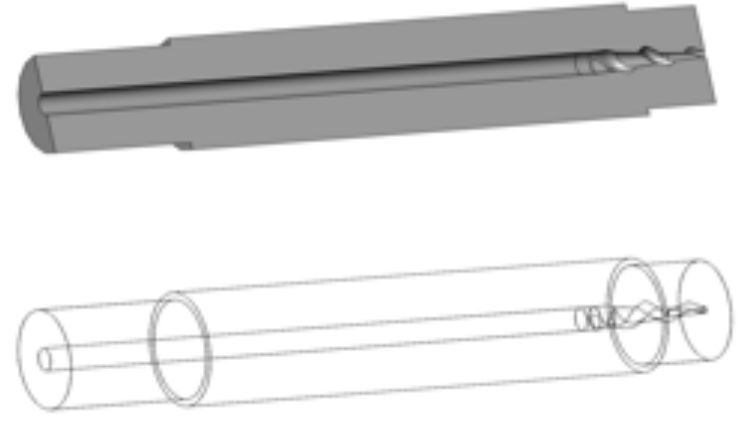

Fig. (1). 3D structure of helix nozzle (S. Zhang et al., 2015).

\section{MATERIALS AND METHODS}

\subsection{Simulation Model of the Helix Nozzle with the Cleaning Target}

To precisely describe the high-pressure water jetting performance of the helix nozzle, and save on experiment- 
associated costs, a simulation approach to build a helix nozzle cleaning process is developed by CFD.

A simulation model derived by $\mathrm{S}$. Zhang et al. is used to study the cleaning performance of the helix nozzle, after which the computational domain and structured grid system is created in GAMBIT. To capture the cleaning process and better forecast velocity and dynamic pressure, a standard target plate is added in front of the nozzle outlet in the computational domain. The size of the target plate refers to the proportion of the nozzle and target plate in industrial production. The jetting motion from the helix nozzle is slightly different in air than is the traditional cylindrical contracting nozzle, which is the optimized resource of the helix nozzle. Jetting from cylindrical contracting nozzle sprays conifies the shape and hits the plate surfaces, effecting the cleaning process via positive pressure. In comparison, jetting from the helix nozzle performs intricately, including a positive force on the plate surface and a shear action caused by the spiral line inside of the helix nozzle. Flow of jet motion out of nozzle is simulated in $3 \mathrm{D}$ space. Regarding the fluid studied as the incompressible Newton fluid, the specified target distance is in the main region, as shown in Fig. (2), and is in accord with S. Zhang et al.'s simulation results.

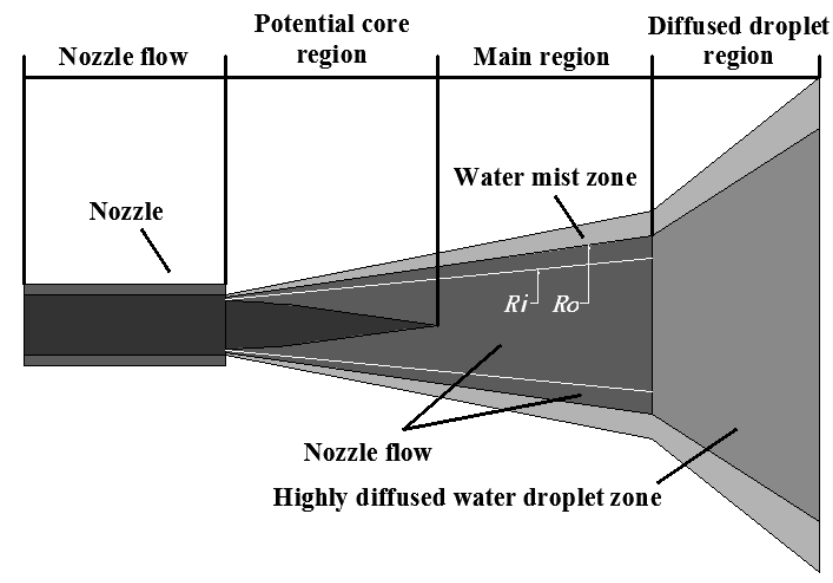

Fig. (2). Jet structure based on theory and observation (Leu et al., 1998).

In the potential core region, which is closed to the nozzle outlet, the differential pressure produced by instable continuous flow stream on the tangential surface could cause eddies. A single spiral line added in the nozzle outlet makes the continuous flow more complex and difficult to describe using an existing simplex theoretical model. The eddy development is dependent on flow velocities, including axial and tangential directions. The water jet inside the potential core region, which is surrounded by a mixing layer, has two main flow directions: Partly rotating it forward follows the single spiral line at the outer water jet layer, and partly rotating it backward drives the flow in a cylindrical contracting shape at the inter water jet layer. Hence, the motion is composite when the water jet sprays out of the nozzle. What is more, the eddy brings about an exchange of mediums such as mass, momentum, constituents, and heat. The composite velocity inside the potential core region is approximate to the jetting velocity at the nozzle outlet at the very moment of spraying.

\subsection{Analysis of the High Pressure Water Jet Cleaning Process}

The damage and failure mode of the fouling layer on a material surface are determined by many factors, for example, glutinosity, mechanical properties, the particle size of the fouling layer, the binding force between the material and fouling layer, and the solid-liquid coupling problem under the dynamic loading function of the water jet. It is difficult to truly describe the cleaning mechanism. Currently, theories and conclusions are gained from abstraction by observing the experiments and analyzing their results. Considering the distinctions in priority and emphasis, different simplifications and postulated conditions lead to different conclusions.

Cleaning of the breakable fouling layer is normally completed via the jet impact effect, which causes local fouling layer formation of flowage and fracture zones. Functions of jet penetration and infiltration bring about the propagation of a fouling layer crack. Subsequently, the continuous jet directly compresses and shears like a water hammer to make the fouling layer rimous, disrupted, and desquamated. Finally, the detached fouling layer is washed away by jet shear action and wash action. The determinant in the breakable fouling layer cleaning process is the pressure acting on fouling layer particles caused by the jet infiltration.

Cleaning of the soft fouling layer is normally completed via shear action, that is, the crushing stress caused by direct contraction and shearing via high pressure. When the stress exceeds the ultimate strength of the fouling layer while increasing according to a certain rule, the high-speed water jet forms a shear layer, which increases quickly because of its instability, and then the shearing layer forms a vortex, which produces strong entrainment on nearby issues. Cracks and fissures are formed on the fouling layer surface and become pits under the continuous water wedging function and are finally washed away. Dynamic pressure, an important factor that measures cleaning ability, regularly attenuates in the main region along the axial line. The property is described as follows [5]:

$\frac{P_{m}}{P_{0}}=\frac{X_{c}}{X}$

where $P_{m}$ is the dynamic pressure on the cross section of the water jet, $P_{0}$ is the dynamic pressure at the nozzle outlet, $X_{c}$ is the jet length within the potential core region, and $X$ is the axial length away from the nozzle outlet. The jet structure and the described equation above shows that the kinetic energy, velocity, and dynamic pressure of the moving jet will decrease due to air friction. While standoff distance increases, cleaning width expansion increases, and jet power loss simultaneously increases. Conversely, decreasing the standoff distance will decrease the cleaning area per unit time.

We have confirmed that the flow model is turbulent. Our case is a constant property fluid flow, indicating that flow density is constant, and that body force in the equation can be neglected. The momentum equations employed are given as follows [6]: 
$\frac{\partial u}{\partial t}+u \frac{\partial u}{\partial x}+v \frac{\partial u}{\partial y}=-\frac{1}{\rho} \frac{\partial p}{\partial x}+v \frac{\partial^{2} u}{\partial x^{2}}+v \frac{\partial^{2} u}{\partial y^{2}}$

$\frac{\partial v}{\partial t}+u \frac{\partial v}{\partial x}+v \frac{\partial v}{\partial y}=-\frac{1}{\rho} \frac{\partial p}{\partial y}+v \frac{\partial^{2} v}{\partial x^{2}}+v \frac{\partial^{2} v}{\partial y^{2}}$

where $v$ is the kinematic viscosity $(v=\mu / \rho)$, consecutively the $v_{T}$ appearance in following equations indicates kinematic turbulent viscosity. The non-conservative governing equations are shown as follows:

$\frac{\partial u}{\partial x}+\frac{\partial v}{\partial y}=0$

$\frac{\partial u}{\partial t}+u \frac{\partial u}{\partial x}+v \frac{\partial u}{\partial y}=-\frac{1}{\rho} \frac{\partial p}{\partial x}+\frac{\partial}{\partial x}\left[\left(v+v_{\mathrm{T}}\right) \frac{\partial u}{\partial x}\right]+$

$\frac{\partial}{\partial y}\left[\left(v+v_{\mathrm{T}}\right) \frac{\partial u}{\partial y}\right]+\frac{\partial}{\partial x}\left[\left(v+v_{\mathrm{T}}\right) \frac{\partial u}{\partial x}\right]+$

$\frac{\partial}{\partial y}\left[\left(v+v_{\mathrm{T}}\right) \frac{\partial v}{\partial x}\right]$

$\frac{\partial v}{\partial t}+u \frac{\partial v}{\partial x}+v \frac{\partial v}{\partial y}=-\frac{1}{\rho} \frac{\partial p}{\partial y}+\frac{\partial}{\partial x}\left[\left(v+v_{\mathrm{T}}\right) \frac{\partial v}{\partial x}\right]+$

$\frac{\partial}{\partial y}\left[\left(v+v_{\mathrm{T}}\right) \frac{\partial v}{\partial y}\right]+\frac{\partial}{\partial x}\left[\left(v+v_{\mathrm{T}}\right) \frac{\partial u}{\partial y}\right]+$

$\frac{\partial}{\partial y}\left[\left(v+v_{\mathrm{T}}\right) \frac{\partial v}{\partial x}\right]$

$\frac{\partial T}{\partial t}+u \frac{\partial T}{\partial x}+v \frac{\partial T}{\partial y}=\frac{\partial}{\partial x}\left[\left(\frac{v}{P r}+\frac{v_{T}}{P r_{T}}\right) \frac{\partial T}{\partial x}\right]+$

$\frac{\partial}{\partial y}\left[\left(\frac{v}{P r}+\frac{v_{T}}{P r_{T}}\right) \frac{\partial T}{\partial y}\right]$

The term $\operatorname{Pr}$ (Prandtl number) is defined by $\operatorname{Pr}=v / \alpha$, where $\alpha$ denotes the molecular diffusivity of heat and $P r_{T}=v_{T} / \alpha$. In addition, the standard $k-\varepsilon$ model equations, expressed in non-conservation form, cause an increase in demand.

$\frac{\partial k}{\partial t}+u \frac{\partial k}{\partial x}+v \frac{\partial k}{\partial y}=\frac{\partial}{\partial x}\left(\frac{v_{T}}{\sigma_{k}} \cdot \frac{\partial k}{\partial x}\right)+$

$\frac{\partial}{\partial y}\left[\frac{v_{T}}{\sigma_{k}} \cdot \frac{\partial k}{\partial y}\right]+P-B$

$\frac{\partial \varepsilon}{\partial t}+u \frac{\partial \varepsilon}{\partial x}+v \frac{\partial \varepsilon}{\partial y}=\frac{\partial}{\partial x}\left(\frac{v_{T}}{\sigma_{\varepsilon}} \cdot \frac{\partial \varepsilon}{\partial x}\right)+$

$\frac{\partial}{\partial y}\left(\frac{v_{T}}{\sigma_{\varepsilon}} \cdot \frac{\partial \varepsilon}{\partial y}\right)+\frac{\varepsilon}{k}\left(c_{\varepsilon 1} P-c_{\varepsilon 2} B\right)$ where the destruction term $B$ is determined by $\varepsilon$, and the production term $P$ is formulated as

$P=2 v_{T}\left[\left(\frac{\partial u}{\partial x}\right)^{2}+\left(\frac{\partial v}{\partial y}\right)^{2}\right]+v_{T}\left(\frac{\partial u}{\partial y}+\frac{\partial v}{\partial x}\right)^{2}$

When the high-speed water jet arrives at the fouling layer, velocity quickly decreases due to momentum transition. Part of the kinetic energy spreads to the fouling layer by way of the stress wave. The high-speed water jet, which transforms while impacting the fouling layer surface, is described by the Mie-Gruneisen equation:

$$
p=\frac{\rho_{0} C^{2} \mu\left[1+\left(1-\frac{\gamma_{0}}{2}\right) \mu-\frac{\beta}{2} \mu^{2}\right]}{\left[1-\left(S_{1}-1\right) \mu-S_{2} \frac{\mu^{2}}{\mu+1}-S_{3} \frac{\mu^{3}}{(\mu+1)^{2}}\right]^{2}}+\left(\gamma_{0}+\beta \mu\right) E
$$

where $p$ is the material pressure, $C$ is the intercept of $u_{s}-u_{p}$ curve, in which $u_{s}$ is spread velocity of stress wave, $u_{p}$ is the kinematic velocity of the particle, $S_{1}, S_{2}$, and $S_{3}$ are the slope coefficients of the $u_{s}-u_{p}$ curve. $\rho_{0}$ is initial density, $\gamma_{0}$ is Gruneisen constant, $\beta$ is the first order volume correction of $\gamma_{0}, E$ is internal energy, and $\mu=(1 / V)-1$, in which $V$ is relative volume.

\section{RSULTS AND DISCUSSION}

We set the boundary conditions for the helix nozzle model simulation, as shown in Table $\mathbf{1 .}$

Table 1. Values of nozzle structure parameters and boundary conditions.

\begin{tabular}{|c|c|c|}
\hline Parameter & Value & Unit \\
\hline \hline Inlet Pressure & $1.0 \times 10^{8}$ & $\mathrm{~Pa}$ \\
\hline Outlet Pressure & 101325 & $\mathrm{~Pa}$ \\
\hline Water Proportion & 9.81 & $\mathrm{kN} / \mathrm{m}^{3}$ \\
\hline Nozzle length & 78 & $\mathrm{~mm}$ \\
\hline Inlet Radius & 1.6 & $\mathrm{~mm}$ \\
\hline Outlet Radius & 0.4 & $\mathrm{~mm}$ \\
\hline
\end{tabular}

As the water jet impacts the fouling layer surface, the velocity and direction will obviously be affected, including momentum and dynamic pressure. The energy loss will be transmitted to the fouling layer. The total force in the impacting process cannot directly represent the damage ability in cleaning the fouling layer. Dynamic pressure, the acting force per unit area functioning on the fouling layer, plays a critical role. A water jet issuing from a traditional nozzle with an axisymmetric structure flows to its 
surroundings with a conical appearance; however, water jet issuing from the helix nozzle is distinctively different. With the action of the spiral line inside, the velocities in the axial center line and near the pipe wall are not symmetrical with respect to the axial center line, nor is dynamic pressure. As shown in Fig. (3), asymmetrical velocity and dynamic pressure distribution in a helix nozzle results in an uneven distribution on the fouling layer surface.

(a) Velocity magnitude distribution in cleaning process

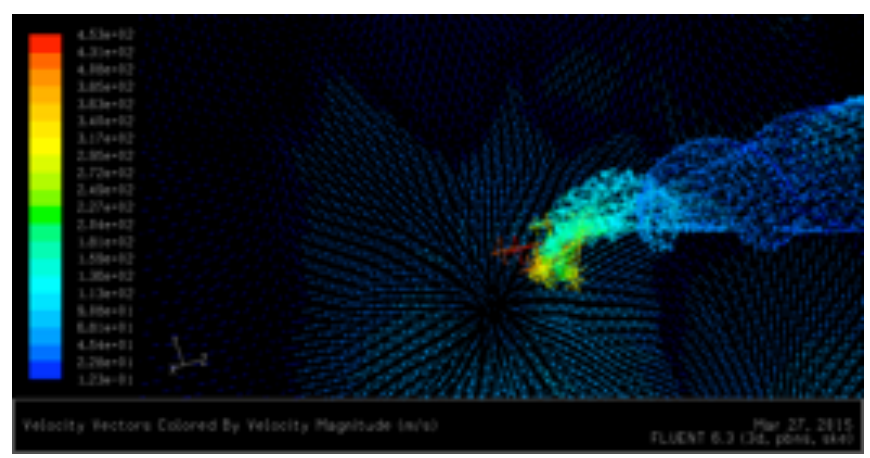

(b) Dynamic pressure distribution in cleaning process

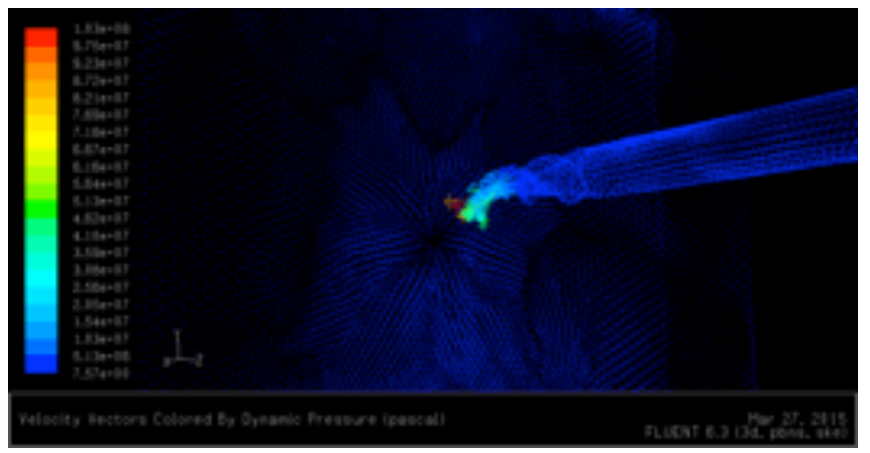

Fig. (3). Velocity magnitude and dynamic pressure distribution in the cleaning process.

The helix nozzle's length was set at $78 \mathrm{~mm}$, as shown in Fig. (4), and the jet velocity and dynamic pressure in the nozzle is in the range of $X=0 \sim 78$ along the axial direction. The area out of the range is the distribution zone out of the nozzle. Every discrete point represents the velocity magnitude of a certain point on a cross section, as shown in Fig. (4a). Velocity magnitude distribution in the forebody of the helix nozzle widely varies, and varies intensively in the later part, considering the contracting configuration. Generally, the velocity magnitude dispersedly distributes outside of the traditional cylindrical contracting nozzle, and the dispersion characteristic is stronger than the distribution inside of the nozzle. However, the jet issuing from the helix nozzle in our study model shows a relatively concentrated distribution of the velocity magnitude, considering the guidance and disturbance by the spiral line. Fig. (4b) shows that the dynamic pressure displays similar characteristics as those shown in Fig. (4a).

Obviously, the velocity out of the helix nozzle is completely different from the traditional cylindrical contracting nozzle, as shown in Fig. (5). Both axial velocity and tangential velocity unevenly diverge from the fouling layer center, and different shades of color distribution were (a) Velocity magnitude in and out of the helix nozzle

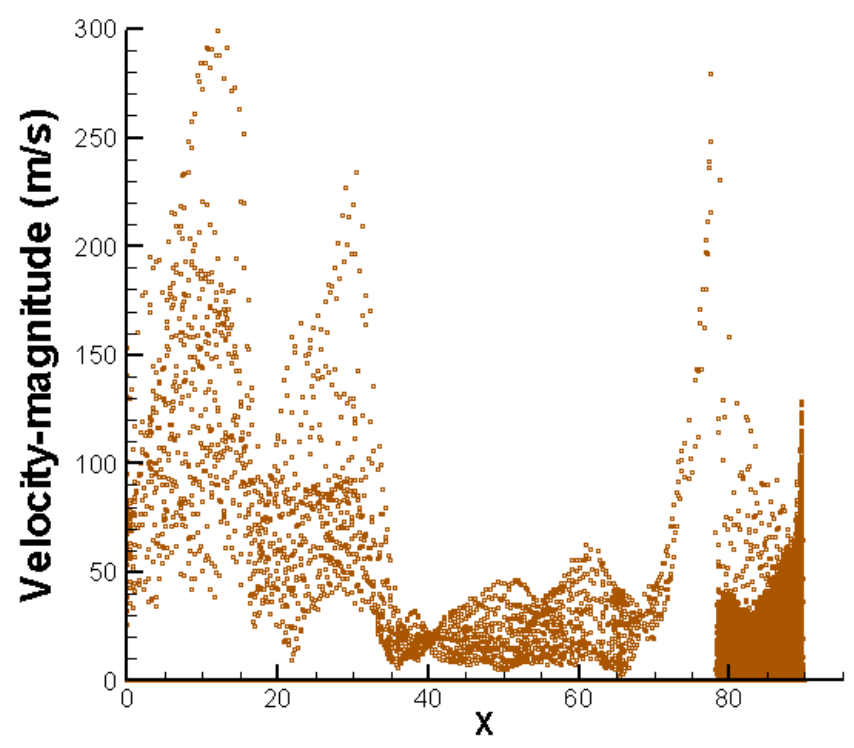

(b) Dynamic pressure in and out of the helix nozzle

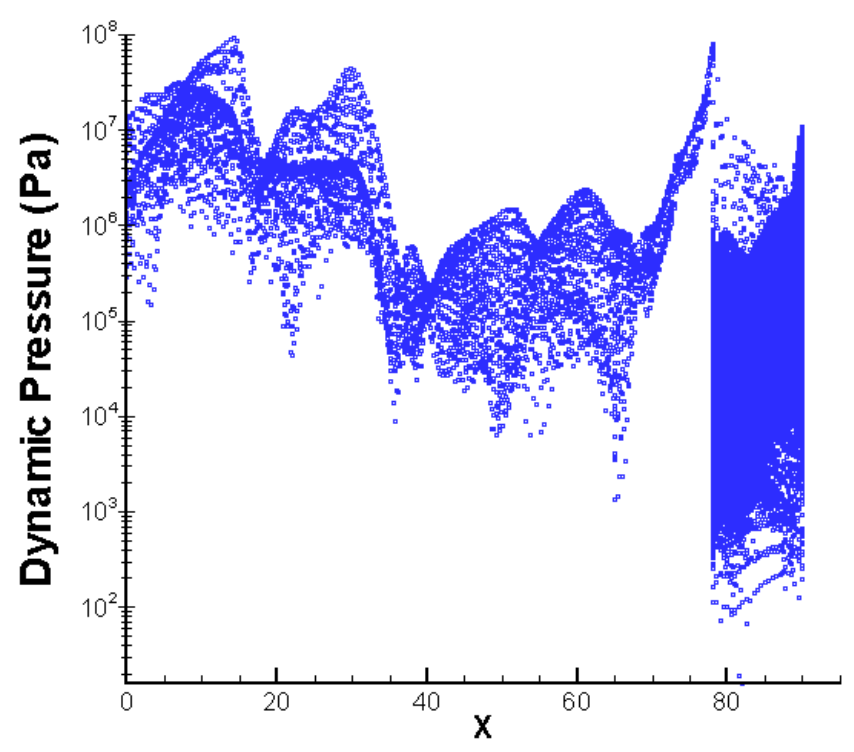

Fig. (4). Jet velocity magnitude and dynamic pressure in and out of the helix nozzle.

observed. Therefore, instead of evenly impacting the fouling layer, the water jet with a shearing action inside generates strong turbulence in the entire jet, the maximum turbulence intensity appears near the boundary of axial jetting, and jetting is guided by the spiral line and the boundary of the environment medium and jet medium. The large-sized shear eddy that forms has a strong entrainment effect on the fouling layer. The fouling layer could be broken by the shearing force generated in the shear eddy flow process. High dynamic pressure is also produced in the interspace of the fouling layer via the continuous shearing jet. Then the fouling layer structure connection ulteriorly weakens and the entire fouling layer finally is peeled away from the material surface via the jet impact action. 
(a) Contours of axial velocity vectors on the fouling layer

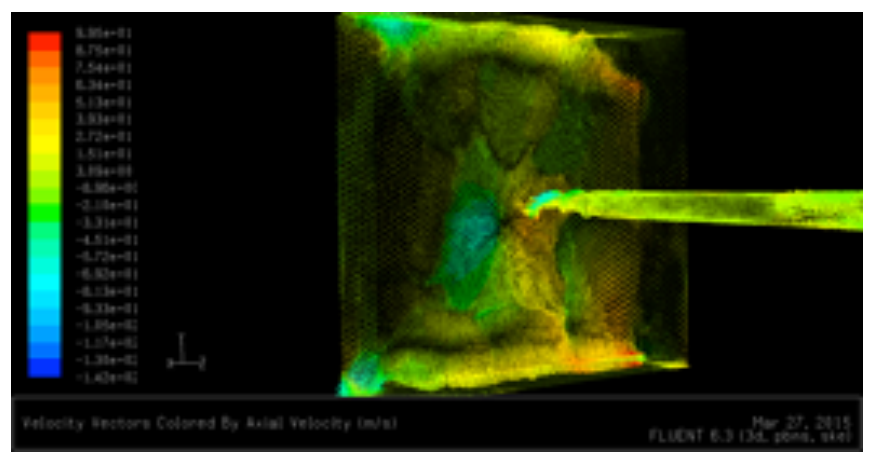

(b) Contours of tangential velocity vectors on the fouling layer

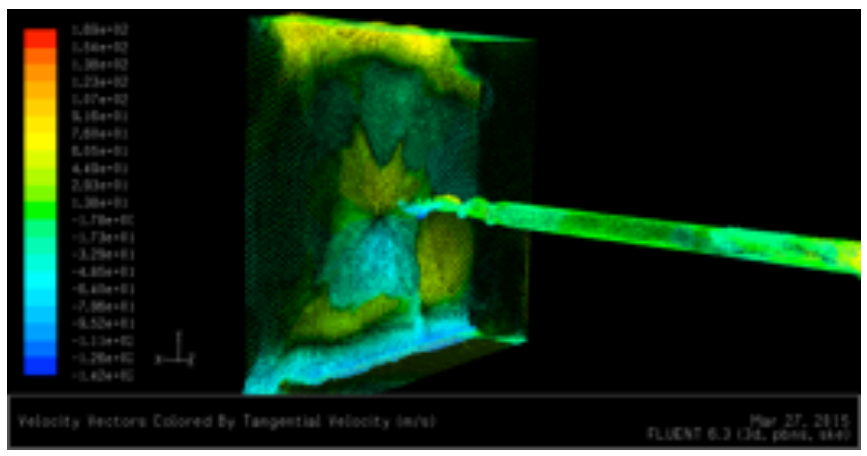

Fig. (5). Contours of velocity vectors on the fouling layer.

\section{CONCLUSION}

This paper presents a theoretical description of the high pressure water jet cleaning process using a particular nozzle model and describes an effective simulation of the cleaning process using this nozzle model. The superiority of the helix nozzle model in the cleaning process was analyzed. The jet issuing from the helix nozzle, which is optimized from the traditional cylindrical contracting nozzle, shows different performance under the guidance of the spiral line. The shearing action of the tangential jetting and the entrainment effect of the shear eddy strengthen cleaning effectiveness.
In future studies, it is necessary to comprehensively discuss the flow characteristic of the helix nozzle, and theoretically describe it, as the formulation is currently unclear because of the difficulty involved in experimenting or simulating jetting performance.

\section{CONFLICT OF INTEREST}

The authors confirm that this article content has no conflict of interest.

\section{ACKNOWLEDGMENTS}

The authors acknowledge the financial support from Dalian Modern Auxiliary Machines Development and Manufacturing Co. and the purely technical help provided by Zhiyuan Jin from Dalian Polytechnic University and Pengtao Yi from Dalian Jiaotong University.

\section{REFERENCES}

[1] M. C. Leu, P. Meng, E. S. Geskin, L. Tismeneskiy, 1998 Mathematical modeling and experimental verification of stationary waterjet cleaning process. Journal of Manufacturing Science and Engineering 120 (3), 571-579.

[2] L. Gensheng, H. Zhongweia, N. Jilei, C. Riji, X. Wei, 2007. The productivity-enhancing technique of deep penetrating perforation with a high-pressure water jet. Petroleum Science and Technology 25 (3), 289-297.

[3] Guha, A., Barron, R.M., Balachandar, R., 2011. An experimental and numerical study of water jet cleaning process. Journal of Materials Processing Technology 211, 610-618.

[4] S. Zhang, X. Tao, J. Lu, X. Wang, Z. Zeng, 2015. Structure optimization and numerical simulation of nozzle for high pressure water jetting. Advances in Materials Science and Engineering.

[5] J. Lu, J. Chen, 2004.Selection for cleaning parameters based on distribution of high-pressure water jet. China Safety Science Journal 14 (12), 67-70

[6] Launder, B. E. \& D. B. Spalding (1974), The numerical computation of turbulent flows, Computer Methods in Applied Mechanics and Engineering 3 (2), 269-289. 